\title{
Incidence of Primary Exercise Headache in Resistance Trained
}

\section{Athletes}

\author{
Pardis Noormohammadpour, ${ }^{1,2}$ Masih Shafiei, ${ }^{1}$ Mohammad Hosein Pourgharib Shahi, ${ }^{1}$ Mohsen
}

Rostami, ${ }^{3}$ Maryam Akbari-Fakhrabadi, ${ }^{1}$ Amir Hossein Memari, ${ }^{1}$ Mohammad Ali Mansournia, ${ }^{4}$ Ehsan

Ghadimi, ${ }^{1}$ Ehsan Sadeghian, ${ }^{1}$ and Ramin $\operatorname{Kordi}^{1,2,}$,

${ }^{1}$ Sports Medicine Research Center, Neuroscience Institute, Tehran University of Medical Sciences, Tehran, IR Iran

${ }^{2}$ Department of Sports and Exercise Medicine, Imam Khomeini Hospital Complex, School of Medicine, Tehran University of Medical Sciences, Tehran, IR Iran

${ }^{3}$ Department of neurosurgery, Shariati hospital, Tehran University of medical sciences, Tehran, IR Iran

${ }^{4}$ Department of Epidemiology and Biostatistics, School of Public Health, Tehran University of Medical Sciences, Tehran, IR Iran

"Corresponding author: Ramin Kordi, Associate Professor of Sports and Exercise Medicine, Sports Medicine Research Center, Tehran University of Medical Sciences, No 7, Al-e Ahmad St., Tehran, IR Iran. Tel: +98-2188630227-8, Fax: +98-2188003539, E-mail: ramin_kordi@tums.ac.ir

Received 2017 March 19; Revised 2017 August 05; Accepted 2017 August 26.

\begin{abstract}
Objectives: The aim of this study was to examine the incidence and patterns of primary exercise headache (PEH) in resistance trained male athletes. PEH is observed particularly during or after severe physical exercise and lasts up to 48 hours. While the focus of the literature has mainly been on the prevalence of PEH in the general population, few studies on athlete populations have been conducted so far. Therefore in a cohort study we examined the incidence and patterns of PEH in resistance trained athletes in an Iranian male sample.

Methods: A population of 116 resistance trained athletes, between 14 to 63 years old, was recruited. PEH was identified in the athletes according to a questionnaire based on the 3rd international classification of headache disorders. The athletes were followed up for six months on a monthly basis while the incidence, pattern and risk factors of PEH were evaluated in them in a precise manner.

Results: The incidence of PEH in overall participants was evaluated 4.8 in 1000 hours exercise. The prevalence of headache during the follow-ups was $19.8 \%$ in which the highest headache incidence occurred in $20-30$ years old athletes. PEH mainly occurred after sports and the quality of PEH was mainly a sudden (81\%) pulsating (47\%) headache which was felt mostly in the occipital region (39\%). The incidence of PEH had a strong statistical correlation with having a history of PEH (odds: 3.8; 95\% CI: 1.02 - 14.3).

Conclusions: Our study showed that incidence and prevalence of PEH in resistance trained athletes might be higher than general population. History of PEH was the main risk factor for developing PEH in resistance trained athletes.
\end{abstract}

Keywords: Benign Exertional Headache, Primary Exertional Headache, Resistance Training, Weight Lifting

\section{Background}

A relatively uncommon type of headache which is caused by some form of exercise without any intracranial disorder is called primary exercise headache (PEH) (1). According to the 3rd edition of the international classification of headache disorders (ICHD-III), PEH occurs solely during or after severe physical exercise and lasts within 48 hours (1).

The literatures' focus was mainly on the prevalence of PEH on the general population over the past decades (2-4). Chen et al. in 2009, studied the prevalence and characteristics of PEH among 1963 Taiwanese adolescents (13 - 15 years old) and $10.2 \%$ of the students met the ICHD-II criteria (3). Furthermore, in the study of Rabiee et al. the prevalence of PEH in Tehran's (Iran) population on a sample size of 2,300 people with a mean age of 36.3 was evaluated 7.3\% (4). How- ever, there has been different findings; for example Pascual et al. in a 10-year study (1996 - 2008) attempted to examine the prevalence and also clinical features of different types of headache in a 6,412 population study and only 11 cases reported $\mathrm{PEH}(0.1 \%)$ (5). The inconsistency in the reported prevalence may be due to recall bias or the characteristics of the study populations (6).

Although examining specific groups (e.g., athletes) for $\mathrm{PEH}$ is merited, few such studies on athlete populations have been conducted so far $(2,7,8)$. In a recent online survey among 14,131 cyclists, 26\% reported PEH which suggests its high prevalence among athletes (2). Furthermore in a case series of 129 athletes with sports-related headache, effortexertion headache was the most prevalent (60\%) type of headache in the none traumatic group which was followed by sports migraine (15\%) headache (7). Therewith, in another study of Australian footballers, the prevalence of mi- 
graine headache complied with the exact IHS definition was estimated $22 \%$ which compared with a community control population (matched by sex and age) in the playing season; was higher (OR: 6.3) (8). There are other small sample size studies on headache in swimmers and basketball players that reported the characteristics of PEH in their samples (9-11).

To the significance of this study, one of the sports which may expose athletes to PEH is resistance training (12). Weight training has been reported as the second most frequent headache-related sports after running (12). The study of Jahani et al. on an Iranian population showed that wrestlers had a higher rate of headache prevalence than other athletes (13). Only a few case reports of headache in weightlifters have been reported so far (1416). Furthermore, previous studies describe three major headache types which are of concern in weightlifters: Benign PEH, cervicogenic headache, and effort-induced migraine headache (12). However, the pathophysiology of the headache is not recognized fully. Mechanisms referred to the disorder are dysfunction of trigeminovascular, cervicogenic muscle tension and incompetence of jugular veins (17). Also, overextended activity can raise intracranial venous sinus pressure, resulting in elevated intracranial pressure, which consequently decreases cerebral blood flow and leads to PEH in weight lifter athletes (12). These studies only form the basis of our understanding of headaches in resistance trained athletes (12). Even in the ICHD-III classification, sub-groups such as weightlifter's headache are mentioned but have not been specifically classified (1). Furthermore, no study has been done on other resistance athletes such as bodybuilders and powerlifters, thus further research is warranted in the field.

To our best knowledge, no epidemiologic study about the incidence, prevalence, characteristics and risk factors of PEH in resistance trained athletes has been published so far. In order to extend the knowledge in this field, in a cohort study, we evaluated the incidence and patterns of PEH in weightlifters, powerlifters and bodybuilders in a sample of Tehran (Iran) sportsmen.

\section{Methods}

\subsection{Participants}

In this short-term cohort study, a sample of 116 men took part. We obtained a list of all the strength training clubs (weightlifting, powerlifting and body building) throughout Tehran (Iran) from the weightlifting federation and contacted their athletes.
The inclusion criteria for the participants were: residence in Tehran (Iran), age between 14 to 63, and athletes who trained more than ten hours per week.

The exclusion criteria for the participants were as follows:

I. Having a history of chronic diseases

II. Not being interested in continuing the study

III. Not answering the telephone at all for different reasons such as phone number release, wrong or blocked phone number, switched off phone or phone line being busy

IV. Being temporarily inaccessible via telephone for at least 2 follow-ups (Temporarily inaccessible means not being able to answer phone calls for two consecutive days)

V. Quitting sports permanently (permanently quitting sports means not to have done sports in a whole month, or when the recession time is unknown or is more than the time left from the follow ups)

The process of this study was confirmed by the ethics committee of Tehran University of Medical Sciences. The participants became aware of the aim and the method of the study in a written form. A written informed consent was taken from the athletes in order to participate in the study.

\subsection{Measures}

General demographic questions (age, weight, height, type of sport, history of headache) were obtained from the participants via an interview. In order to discover the prevalence, pattern and risk factors of PEH, a Persian questionnaire was used. The questionnair is based on the international headache society (IHS) criteria of ICHD-III and a previous study which was designed to study PEH in the general population (4). Misunderstanding Index was assessed in a pilot study and none of items got a misunderstanding index $>20 \%(18,19)$. Also content validity of the questionnaire was evaluated through content validity index (CVI) by the members of an expert panel and CVI was equal to the essential value (1.00) for all questions (20). After the questionnaire's validity was approved, it was applied in data collection. According to ICHD-III the diagnosis of PEH was based on the following criteria (1):

"A. At least two headache episodes fulfilling criteria B and $\mathrm{C}$

B. Brought on by and occurring only during or after strenuous physical exercise

C. Lasting $<48$ hours

D. Not better accounted for by another ICHD-III diagnosis".

Furthermore, the questionnaire included differential diagnoses of migraine, tension type headache and posttraumatic headache. This questionnaire was adjusted ac- 
cording to different features of the three sports (bodybuilding, weightlifting and powerlifting). It consisted of 54 questions which were divided into three parts and answers were scored and analyzed at the end of the study.

In the first part (19 questions), the volunteers were asked about their history of migraine and tension headaches according to ICHD-III. As they are the most typical headaches in the general population, the relation between these two types of headaches and PEH was evaluated. The questions asked about the onset age of their headache, the exact region of pain, type of headache (e.g., pulsating or headache attack), the triggering factors (e.g., coughing, physical activity, sport, sneezing, sexual activity, thirst or voiding), the time interval between exposure to the triggering factors and onset of headache, and other side effects (nausea, vomiting, photophobia, phonophobia).

In the second part (7 questions) the athletes were asked about their history of post-traumatic headache and its relation to PEH. The questions included: how and when the trauma occurred and whether any dysfunctions like unconsciousness or short term amnesia occurred.

In the third (and last) part (28 questions), the athletes were asked about their history and pattern of PEH. The questions included the type of sports causing headache, position of sports (neck movement, warming up etc.), the time of experiencing headache during sports, the region in which pain was felt, description of the pain (constant, stabbing, burning, or vague pain etc.), factors worsening the headache (altitude, hot weather, menstruation, specific food). Whether PEH occurred concurrently with migraine or tension type headache was also asked in the questionnaire. In addition, in the third part a Visual Analog Scale (VAS) was applied to assess the severity of pain during or after exercise. Our VAS was a $10 \mathrm{~cm}$ solid horizontal line which was marked from left (0) to right (10). The participants were asked to imagine the most intense pain they had experienced in their life as number 10 and according to that, signify how much pain they had experienced in sport choosing on a number.

\subsection{Procedure}

A researcher interviewed the participants face to face and filled in the questionnaire. After filling out the questionnaire and collecting basic information from the participants, the athletes were followed up for five months on a monthly basis. The athletes were interviewed with a phone call on each follow-up point by the same researcher. Consequently, the severity, pattern and the risk factors of the headache in a specified time interval were obtained precisely. Also, in the follow-up, the number of exercise sessions and the time spent in every session were asked.
Then the PEH incidence and its pattern were evaluated by the third part of the questionnaire. To record features of headache attack, a questionnaire was filled in for each one.

\subsection{Statistics}

To analyze the quantitative data, mean (SD) were utilized. Categorical data were demonstrated by number (percentage). The response rate in each follow-up was calculated by dividing number of participants who picked up their phone and answered our telephone call (whom exercised more than 10 hours per week) by all participants in that follows up. The incidence of PEH is reported by number of headaches in 1000 hours of practice. For estimating the risk factors of PEH, the statistical tests, Multi-level Mixed effect logistic regression were used and the results were presented as odds ratio. The significance of the tests was determined as P value $<0.05$. The statistical calculations of the study were measured with SPSS software, version 19 (SPSS Inc, Illinois, USA).

\section{Results}

One hundred and sixteen athletes entered the study. Figure 1 shows the response rate and follow-up periods of athletes. The demographic and basic data of the athletes at the beginning of study are presented in Table 1. Also, the distribution of PEH in different resistance sports during follow ups is presented in Figure 2.

\subsection{Prevalence and Incidence of PEH}

During the follow-ups, 38 PEH incidences occurred in 23 participants according to ICHD-III criteria. The prevalence of headache during the follow-ups was $19.8 \%$ which the highest headache incidence occurred in 20 - 25 years old athletes (Figure 3). The incidence of PEH in overall participants was 4.8 per 1000 hours of exercise. Based on fields of sports, it was 4.2 per 1000 hours of exercise among weightlifters, 8 per 1000 hours of exercise in powerlifters and 5.8 per 1000 hours of exercise in bodybuilders. Our study showed that point prevalence of headache among weightlifters was 10 (8.6\%) and in bodybuilders was $1(0.9 \%)$. None of the powerlifters had experienced PEH at the beginning of the study. It is notable that at the start of study, $28.9 \%$ of the participants with PEH had a history of other types of headache.

\subsection{Pattern of PEH}

The pattern of PEH diagnosed by ICHD-III during current study is demonstrated in Table 2. The results from follow-ups showed that PEH mainly occurred after sports compared to during sports (61\% vs. $39 \%$ ). The quality 
Table 1. Basic and Demographic Data of Participants at Onset of Study

\begin{tabular}{|c|c|c|}
\hline Variant & & Participants $N=116$ \\
\hline Age & Mean (SD) & $27.0(10.4)$ \\
\hline Weight, kg & Mean (SD) & $90.0(19.0)$ \\
\hline Height, cm & Mean (SD) & $176.0(12.2)$ \\
\hline \multirow{3}{*}{ Type of sport $^{\mathrm{a}}$} & Weight lifter & $73(62.9)$ \\
\hline & Power lifter & $4(3.4)$ \\
\hline & Body builder & $39(33.6)$ \\
\hline \multirow{2}{*}{ History of headache ${ }^{a}$} & No & $61(52.5)$ \\
\hline & Yes & $55(47.41)$ \\
\hline \multirow{3}{*}{ History of $\mathbf{P E H}^{\mathrm{a}}$} & Few months ago & $13(11.2)$ \\
\hline & More than a year ago & $1(0.9)$ \\
\hline & More than five years ago & $1(0.9)$ \\
\hline \multirow{3}{*}{ History of Migraine $^{a}$} & & $5(4.3)$ \\
\hline & Migraine without aura & $4(3.4)$ \\
\hline & Migraine with aura & $1(0.9)$ \\
\hline $\begin{array}{l}\text { History of Probable } \\
\text { migraine }^{\text {a }}\end{array}$ & & $14(12.0)$ \\
\hline $\begin{array}{l}\text { History of Tension } \\
\text { headache }^{\text {a }}\end{array}$ & & $14(12.0)$ \\
\hline $\begin{array}{l}\text { History of } \\
\text { Post-traumatic } \\
\text { headache }^{\text {a }}\end{array}$ & & $5(4.3)$ \\
\hline $\begin{array}{l}\text { History of } \\
\text { Undifferentiated }^{\mathrm{a}}\end{array}$ & & $2(1.7)$ \\
\hline
\end{tabular}

of PEH in our study was mainly a sudden (81\%) pulsating (47\%) headache which was felt mostly on the occipital region (39\%). The mean (SD) severity of the headache attacks (VAS) was 3 (1.58). Other characteristics of the headache are demonstrated in Table 2. In sixty percent of the PEH occasions, athletes stopped exercising and in forty percent, athletes continued on with their sports. It is notable that athletes report no aura with the PEH.

Further analysis showed that incidence of PEH had a strong association with having a history of PEH (OR: 3.8; 95\% CI: 1.02 - 14.3). Also, the results of a multivariate analysis showed no correlation between headache and the age of the participants (OR: 0.94; 95\% CI: $0.87-1.01$ ). This study showed that the incidence of PEH among weightlifters, powerlifters and bodybuilders had no statistical correlation with having a background of migraine, probable migraine, tension headache and post-traumatic headache (Table 3).

The results of chi square showed $76 \%$ of the athletes

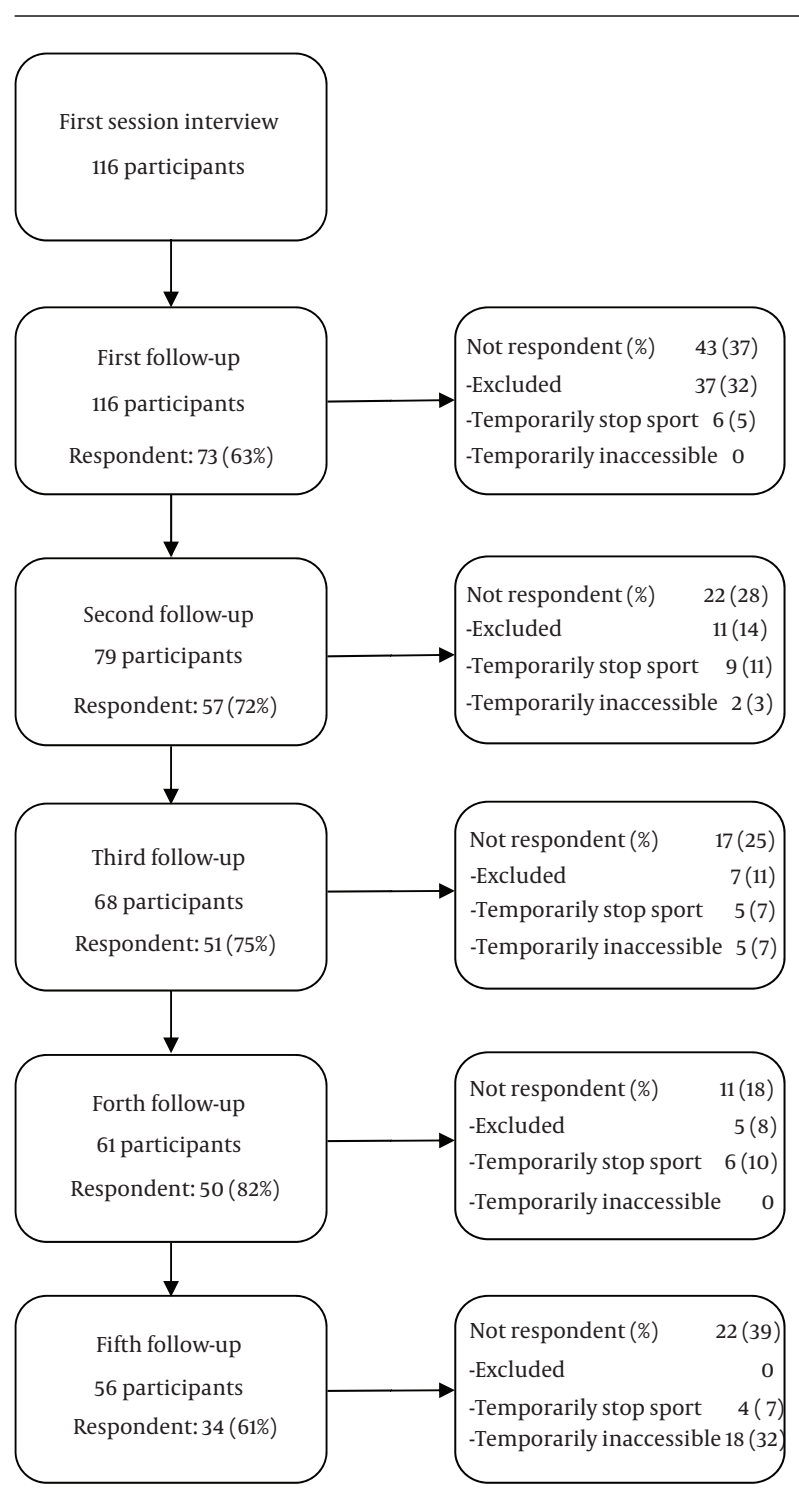

Figure 1. Flowchart of Follow-Ups

experienced PEH while exercising with the upper limbs ( $\mathrm{P}$ $=0.027$ ) while only $24 \%$ of the athletes experienced PEH while training with the lower limb $(\mathrm{P}<0.001)$. Furthermore, neck movement during weightlifting was reported to be related to $\mathrm{PEH}$ in $76 \%$ of the athletes. Other probable related factors to PEH are demonstrated in Table 4. Factors that decreased the severity of PEH were painkillers (34\%) and night sleep (11\%). In addition, drinking sufficient water during exercise, being in a quiet place, darkening the room and halting exercise were reported by other participants ( 2 cases for each item) whose pain was declined. 


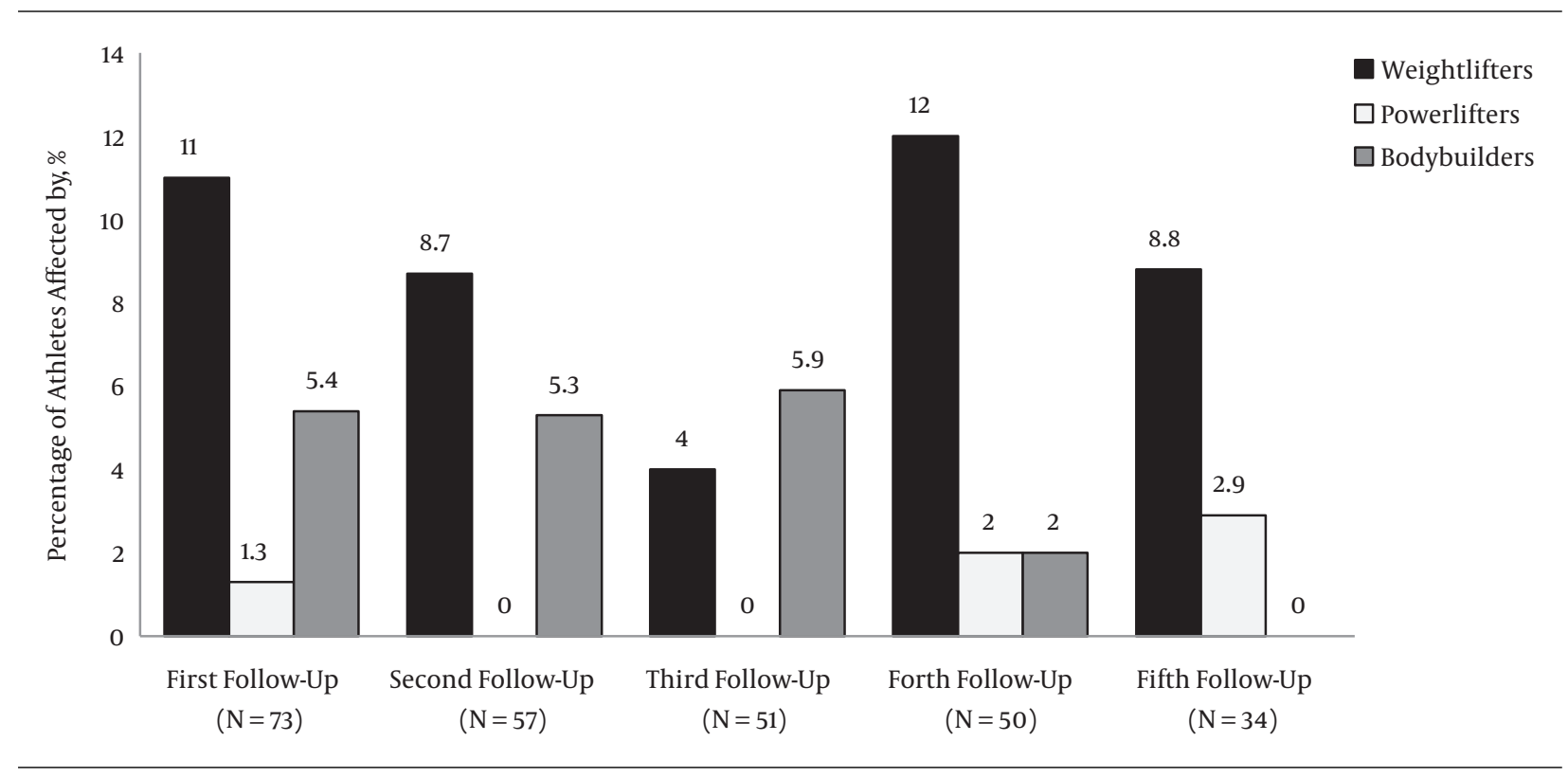

Figure 2. Primary Exercise Headache Distribution in Different Resistance Sports During Follow Ups

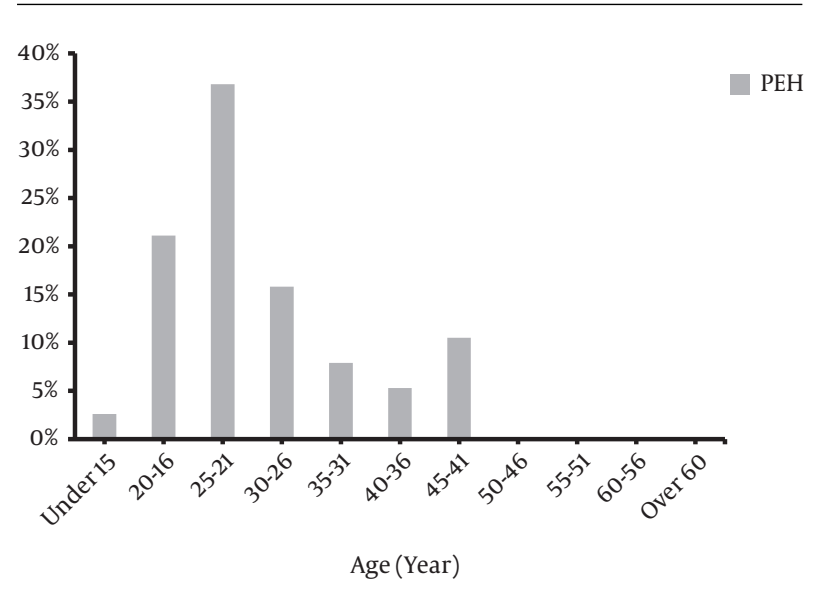

Figure 3. Distribution of PEH Among Different Age Groups

\section{Discussion}

The current study evaluated the incidence and patterns of PEH in a sample of resistance trained athletes. Overall results showed that the incidence of PEH in a 5month follow up was 38 headache events among 23 participants (out of 116 athletes). The incidence rate of PEH in all participants was 4.8 per 1000 hours of exercise with the higher rate in powerlifters.

The prevalence of PEH in this study was $19.8 \%$ which was rather close to the study of cyclists (26\%) (2) and Australian footballers (22\%) (8). However, general population studies showed a lower incidence and prevalence of PEH.
For example, in a prospective study which clarified the incidence of different types of headache in a large-scale general population study, 11 subjects (out of 6,412) with PEH were diagnosed during the 10 years of study (0.1\%)(5). Furthermore, in the general population of Portugal and Denmark, PEH was estimated about $0.2 \%$ and $1 \%$ (21). Also one year prevalence of PEH was reported about 7.3\% in general population of Tehran (Iran) (4). There were also higher rates of PEH reported by Sjaastad et al. (12.3\%) (22) and the study of Chen et al. in adolescents (10.2\%) (3). Comparing the above results, clearly demonstrates the high incidence and prevalence of headache in a sport population compared to a general population. The possible explanation could be due to the characteristics of general population (e.g., being less physically active) (5). Furthermore, adherence to exercise in population based studies was not defined $(2,4,5)$.

To provide more explanation, one can address several underlying mechanisms for headache in sports which make this group more susceptible to headache. In resistance sports, the main mechanisms leading to headache may be categorized in accordance with three main headache types which are common in weightlifters: $\mathrm{PEH}$, effort-induced migraine headache, and cervicogenic headache (CEH) (12). PEH is the type of headache assessed in our study according to ICHD-III. Valsalva maneuver or straining during exercise is a mechanism supposed for $\mathrm{PEH}$ (12). It is suggested that PEH has a vascular nature (12). Overextended activity raises intracranial venous sinus pressure, resulting in elevated intracranial pressure, 
which consequently decreases cerebral blood flow and leads to PEH (12). Furthermore, angiographic studies in patients with PEH have shown multiple areas of segmental cerebral arterial vasospasm $(23,24)$. Previous investigations conducted on weightlifters have shown that the systolic and diastolic pressure may rise as much as 400 and $300 \mathrm{mmHg}$ respectively during maximum weight lifting which could lead to headache (12). This mechanism may explain the lower rate of PEH among bodybuilders and powerlifters compared to weightlifters, as they do not lift very heavy weights. Also, recent data suggested that intracranial venous congestion due to internal jugular venous valve incompetence could play a role in PEH (1). A possible mechanism proposed for effort-induced migraine headaches which is reported in long distance runners is hyperventilation leads to vasoconstriction which in return triggers a reactive vasodilatation and headache $(25,26)$ so this type of headache is less likely to occur in weightlifting athletes (26). Finally, suffering from anatomical abnormalities inside the neck including synovial joints, cervical ligaments and intervertebral discs could lead to CEH type of headache (12).

To address the pattern of PEH, it revealed that the most headache incidences in this study occurred in 20 - 25 years old athletes. This finding is in line with previous case reports in weightlifters which reported headache in the same age group (14-16). This finding was also consistent with studies reported that PEH was more prevalent in the young athletes $(2,7)$. As an explanation to this finding, older athletes generally avoid high-intensity weight shots which may trigger headache; therefore, the age groups suffering from headache are mainly adolescents and young adults. Furthermore, older people are less likely to obtain higher heart rates compared to young athletes (2). However, in contrast to this result, Raymond et al. discovered that old age is a risk factor for developing headache in pool players (27). The finding has been explained by the type of sport. Hyperextension of neck in pool players is a risk factor for headache which (CEH) is more prevalent among older players with degenerative changes in their cervical spine (27). Moreover, in a case series of swimmers, the mean age of 7 athletes suffering from headache was 41 years (9); and they proposed that the candidate responsible mechanism was insufficient ventilation which led to hypercapnia and subsequently produced vasodilation and augmentation in intracranial pressure (9). Furthermore, swimming is a kind of sport which is popular in all age groups, which could explain the prevalence of headache among older ages. By considering the above studies, we can extrapolate that the age group which is more active in a certain sport can affect the prevalence of PEH.

The quality of PEH in our study was mainly a sudden, bi- lateral, pulsating headache felt mostly on the occipital region which mainly occurred during exercise or in the first thirty minutes after exercise. In consistence with our results, several case reports in resistance athletes (14-16) and swimmers (9) have reported a sudden acute headache during or immediately after exercise. Also, most large studies have reported a bilateral feature of $\operatorname{PEH}(3,5,28)$ in line with our findings. Case reports in weightlifters (16), swimmers (9) and also the study held on adolescents showed a pulsating type of PEH (3). It is notable that the pulsating or throbbing characteristic of PEH in some athletes can support the vascular nature of the headache (12). However, in the study of van der Ende-Kastelijn et al. (2), cyclists reported dull and throbbing kinds of headache (42\% and $23 \%$, respectively) which were mainly unilateral. In accordance with our outcomes, headache which was mostly felt in the occipital region has been reported from different case reports of weightlifters (14-16) and also from the study of Japanese population (28). Yet, some studies indicate other regions such as temporal in swimmers (11) or the frontal region (3). The discrepancy between the results of these different studies could be due to variety of studies' population or different sports which the athletes were involved in.

\subsection{Probable Risk Factors or Correlations of PEH}

This study showed that the incidence of PEH had a strong statistical association with having a history of PEH but not with other headache types. Similarly, in another study on pool players, the estimated adjusted odds ratio of previous headaches' history for PEH was about 10.1 (95\% CI: 3.36 - 30.4) (27). Other related factors for PEH were training with upper limbs, the heaviness of the weights, or neck movement. It seems that severe training increases intracranial pressure, which subsequently decreases cerebral blood flow, and results in the development of a headache (12). So the heavier the weights lifted, the blood flow would be the less and the possibility of headache incidence will rise. Furthermore, previous studies have shown that during weightlifting the blood pressure rises which could increase the risk of headache (12). Considering the effect of neck movement and using upper limbs in weight lifting as a risk factor for headache, the pathogenesis may arise from the pressure on neck structures while lifting weights. Since the most pain felt was the occipital region in our athletes, it is probable that the structural elements in neck and sub-occipital region are also involved in the headache.

With respect to the coexistence of PEH and other primary headaches, the present study highlighted the coexistence of other headache types with PEH. The results 
showed at the highest rate, $13.2 \%$ of the athletes experienced tension headaches followed by probable migraine (7.9\%) along with PEH. It is notable that tension headache and probable migraine were the most prevalent headache types among the athletes at the time of beginning the study. Also van der Ende-Kastelijn et al. reported tension headache as the most prevalent headache (23.8\%) along with PEH in cyclists (2). Coexistence of PEH with migraine in our study was low (2.6\%). However, in the study of the Japanese population, 67\% experienced migraine without aura along with PEH (28). According to Chen et al. patients with pre-existing migraine are likely to have PEH characteristics close to migraine, whereas patients suffering from PEH alone mainly experience PEH with fewer migraine type characteristics (3). PEH and migraine are of two different types of headache, even though they have some features in common (3). The presentations of headaches induced by exercise are different from migraine even in patients suffering from migraine (3). By considering the small number of athletes with history of migraine at the start of study (4.3\%), possible features of migraine were less expected along with PEH (2.6\%). The comorbidity of PEH and post-traumatic headache was less prevalent in our study (5.3\%), whereas in other sports such as contact sports a higher rate of post-traumatic headache is seen (29). In the contact sports the higher risk of head strike or collision increases the prevalence of post-traumatic headache; however in our study, the individual and non-contact nature of the assigned sports would be an explanation for the lower prevalence of post-traumatic headache.

To the best our knowledge this is first study which investigates the incidence rate of PEH in resistance trained athletes and the different characteristics and risk factors leading to PEH. The results could help practitioners in applying different interventions in prevention, diagnosis and treatment of PEH. For example, they may implement different relaxation strategies and cervical rehabilitation methods (30) during or after exercise sessions to prevent $\mathrm{PEH}$ in their athletes. It could give researchers further ideas in discovering whether these headaches may influence the maximal performance of the athletes; and which complications can arise in a long-term follow up.

This study also has several limitations. First, the small sample size which made some statistical results at borderline significance (e.g., the relation of age and PEH). Second, we defined all sport related headaches as PEH according to ICHD-III; therefore, lack of categorization for other headache types classified in previous studies (cervicogenic, benign exertional headache and effort-induced migraine) is seen. Third, all the athletes in our study were male and we could not compare the incidence of PEH between two genders. Finally, our study was a questionnaire based study and no objective assessments such as neuroimaging examination were performed.

\section{Conclusions}

This study showed a high rate of incidence and prevalence of PEH in resistance trained athletes compared to existing data from general populations. Having a history of $\mathrm{PEH}$, as the main risk factor, and training with upper limbs and neck movement were other main related factors for developing PEH in resistance trained athletes. Furthermore, tension headache was more accompanied with PEH in resistance trained athletes than other types of headache.

\section{Footnotes}

Authors' Contribution: Mohsen Rostami, Masih Shafiei, Ramin Kordi, and Pardis Noormohammadpour contributed to study conception and design; Mohsen Rostami, Ehsan Sadeghian, Ehsan Ghadimi and Masih Shafiei contributed to acquisition of data; Pardis Noormohammadpour, Ramin Kordi, Amir Hossein Memari and Mohammad Ali Mansournia contributed to interpretation of data; Pardis Noormohammadpour, Amir Hossein Memari, Mohammad Hosein Pourgharib Shahi and Maryam Akbari-Fakhrabadi contributed to drafting of manuscript; Pardis Noormohammadpour, Mohsen Rostami, Masih Shafiei, Maryam Akbari-Fakhrabadi, Amir Hossein Memari, Mohammad Hosein Pourgharib Shahi, Mohammad Ali Mansournia, Ehsan Ghadimi, Ehsan Sadeghian and Ramin Kordi contributed to critical revision. All authors have read and approved the final version of the manuscript, and agree with the order of presentation of the authors.

Funding/Support: This work was supported by Tehran University of Medical Sciences \& health Services grant (8803-53-9434).

Competing Interests: None of the authors declare competing financial interests.

Conflict of Interest: The authors have no conflicts of interest to declare.

Ethics Committee or Institutional Review Board: Review Board and ethics committee of Tehran University of Medical Sciences and health Services. Assigned number: 88-03-53-9434

\section{References}

1. Headache Classification Committee of the International Headache Society (IHS). The international classification of headache disorders. 3rd ed. 33. ; 2013. 
Table 2. Characteristics of Primary Exercise Headache in the Follow-Ups ${ }^{\mathrm{a}}$

\begin{tabular}{|c|c|c|}
\hline $\begin{array}{l}\text { Primary Exercise } \\
\text { Headache } \\
\text { Characteristic }\end{array}$ & & $\begin{array}{l}\text { Headache Incidences } \\
\qquad(\mathrm{N}=38)^{\mathrm{b}}\end{array}$ \\
\hline \multirow{2}{*}{ Headache incidence } & After exercise & $23(61)$ \\
\hline & During exercise & $15(39)$ \\
\hline \multirow{3}{*}{$\begin{array}{l}\text { Time interval } \\
\text { between headache } \\
\text { occurrence and } \\
\text { onset of exercise }\end{array}$} & Less than 30 minutes & $8(53)$ \\
\hline & 30 to 60 minutes & $7(47)$ \\
\hline & One to two hours & - \\
\hline \multirow{3}{*}{$\begin{array}{l}\text { How the headache } \\
\text { starts }\end{array}$} & $\begin{array}{l}\text { Suddenly (in few } \\
\text { seconds) }\end{array}$ & $31(81)$ \\
\hline & $\begin{array}{l}\text { Rapidly (in few } \\
\text { minutes) }\end{array}$ & $6(16)$ \\
\hline & $\begin{array}{c}\text { Gradually (in few } \\
\text { hours) }\end{array}$ & $1(3)$ \\
\hline \multirow{5}{*}{$\begin{array}{l}\text { How long the } \\
\text { headache attack } \\
\text { lasts }\end{array}$} & Less than five minutes & $3(8)$ \\
\hline & Five to sixty minutes & $35(92)$ \\
\hline & One to 24 hours & - \\
\hline & 24 to 48 hours & - \\
\hline & More than 48 hours & - \\
\hline \multirow{7}{*}{ Quality of headache } & Constant pain & $9(24)$ \\
\hline & Stabbing pain & $4(11)$ \\
\hline & Pulsating & $18(47)$ \\
\hline & Radiant & $2(5)$ \\
\hline & Vague pain & $5(13)$ \\
\hline & Sharp pain & - \\
\hline & $\begin{array}{l}\text { Pain which increases } \\
\text { in hot or cold } \\
\text { temperature }\end{array}$ & - \\
\hline \multirow{2}{*}{$\begin{array}{l}\text { Unilateral/Bilateral } \\
\text { pain }\end{array}$} & Unilateral & $13(34)$ \\
\hline & Bilateral & $25(66)$ \\
\hline \multirow{6}{*}{ Headache site } & Occipital & $15(39)$ \\
\hline & Temporal & $9(24)$ \\
\hline & Frontal & $7(18)$ \\
\hline & Vertex & $4(11)$ \\
\hline & Entire head & $2(5)$ \\
\hline & Could not specify & $1(3)$ \\
\hline \multirow{2}{*}{$\begin{array}{l}\text { Intensity of } \\
\text { headache attack } \\
\text { (VAS) }\end{array}$} & Mean (SD) & $3(1.58)$ \\
\hline & Range & $1-8$ \\
\hline \multirow{5}{*}{$\begin{array}{l}\text { PEH along with } \\
\text { previous headaches, }\end{array}$} & Migraine & $1(2.6)$ \\
\hline & Probable migraine & $3(7.9)$ \\
\hline & Tension headache & $5(13.2)$ \\
\hline & $\begin{array}{c}\text { Post-traumatic } \\
\text { headache }\end{array}$ & $2(5.3)$ \\
\hline & Aura & - \\
\hline
\end{tabular}

Abbreviations: N, number, PEH, primary exercise headache; SD, standard devi-

ation.

${ }^{\mathrm{a}}$ Values are expressed as No. (\%)

${ }^{\mathrm{b}}$ According to ICHD-III.
2. van der Ende-Kastelijn K, Oerlemans W, Goedegebuure S. An online survey of exercise-related headaches among cyclists. Headache 2012;52(10):1566-73. doi: 10.1111/j.1526-4610.2012.02263.x. [PubMed: 23126543].

3. Chen SP, Fuh JL, Lu SR, Wang SJ. Exertional headache-a survey of 1963 adolescents. Cephalalgia. 2009;29(4):401-7. doi: 10.1111/j.14682982.2008.01744.x. [PubMed: 19025550].

4. Rabiee B, Mohammadinejad P, Kordi R, Yunesian M. The Epidemiology of Exertional Headache in the General Population of Tehran, Iran. Headache. 2015;55(9):1225-32. doi: 10.1111/head.12610. [PubMed: 26198401].

5. Pascual J, Gonzalez-Mandly A, Martin R, Oterino A. Headaches precipitated by cough, prolonged exercise or sexual activity: a prospective etiological and clinical study. J Headache Pain. 2008;9(5):259-66. doi: 10.1007/s10194-008-0063-5. [PubMed: 18751938].

6. Wang SJ, Fuh JL. The "other" headaches: primary cough, exertion, sex, and primary stabbing headaches. Curr Pain Headache Rep. 2010;14(1):41-6. doi: 10.1007/s11916-009-0083-0. [PubMed: 20425213].

7. Williams SJ, Nukada H. Sport and exercise headache: Part1. Prevalence among university students. BrJ Sports Med. 1994;28(2):90-5. [PubMed: 7921915].

8. McCrory P, Heywood J, Coffey C. Prevalence of headache in Australian footballers. $\mathrm{Br} J$ Sports Med. 2005;39(2):10. doi: 10.1136/bjsm.2004.014860. [PubMed: 15665188].

9. Kim JS. Swimming headache followed by exertional and coital headaches. J Korean Med Sci. 1992;7(3):276-9. doi: 10.3346/jkms.1992.7.3.276. [PubMed:1285928].

10. Kinart CM, Cuppett MM, Berg K. Prevalence of migraines in NCAA division I male and female basketball players. National Collegiate Athletic Association. Headache. 2002;42(7):620-9. [PubMed: 12482214].

11. Mizoguchi K, Utsunomiya H, Emoto H, Shimizu T. Benign exertional headaches induced by swimming. Kurume Med J. 1990;37(4):261-3. [PubMed: 2097442].

12. Rifat SJ. Diagnosis and management of headache in the weight-lifting athlete. Curr Sports Med Rep. 2003;2(5):272-5. [PubMed:12959709].

13. Jahani P, Salesi M, Marzban M, Abdollahifard G. The Prevalence of Headache Among Athletic University Students. Asian J Sports Med. 2016;7(1):33515. doi: 10.5812/asjsm.33515. [PubMed: 27231525].

14. Powell B. Weight lifter's cephalgia. Ann Emerg Med. 1982;11(8):449-51. [PubMed: 7103166].

15. Paulson GW. Weightlifters headache. Headache. 1983;23(4):193-4 [PubMed: 6885412].

16. Ibbotson SH. Weight-lifter's headache. Br J Sports Med. 1987;21(3):138 [PubMed: 3676641].

17. Simpson M. Weightlifter's headache. Pract Neurol. 2016;16(3):215-6. doi: 10.1136/practneurol-2015-001227. [PubMed: 27013484].

18. Lawshe $\mathrm{CH}$. A quantitative approach to content validity. Pers Psychol. 1975;28(4):563-75.

19. Guillemin F, Bombardier C, Beaton D. Cross-cultural adaptation of health-related quality of life measures: Literature review and proposed guidelines. J Clin Epidemiol. 1993;46(12):1417-32. [PubMed: 8263569].

20. Ayre C, Scally AJ. Critical values for lawshe's content validity ratio revisiting the original methods of calculation. Meas Eval Couns Dev. 2013;47(1):79-86.

21. Rasmussen BK, Olesen J. Symptomatic and nonsymptomatic headaches in a general population. Neurology. 1992;42(6):122531. [PubMed: 1603351].

22. Sjaastad O, Bakketeig LS. Exertional headache. I. Vaga study of headache epidemiology. Cephalalgia. 2002;22(10):784-90. doi: 10.1046/j.1468-2982.2002.00466.x. [PubMed: 12485203].

23. Silbert PL, Hankey GJ, Prentice DA, Apsimon HT. Angiographically demonstrated arterial spasm in a case of benign sexual headache 
Table 3. Incidence odds ratio (OR) Estimate the Risk Factors of PEH

\begin{tabular}{lccc}
\hline Variant & OR Crude (CI 95\%) & P Value & OR Adjusted (CI 95\%) \\
\hline Age, $y$ & $0.94(0.87-1.01)$ & 0.08 & $0.94(0.88-1.01)$ \\
History of PEH & $3.8(1.02-14.3)$ & $0.047^{\mathrm{a}}$ & $3.5(0.93-13.3)$ \\
Migraine & $1.07(0.15-7.77)$ & 0.95 & - \\
Probable migraine & $1.89(0.30-12.0)$ & 0.50 & - \\
Tension headache & $1.95(0.58-6.45)$ & 0.28 & - \\
Post-traumatic headache & $2.3(0.16-34.20)$ & 0.54 & - \\
\hline
\end{tabular}

Abbreviations: $\mathrm{CI}$, confidence interval, adjusted for age; $\mathrm{PEH}$, primary exercise headache.

${ }^{\mathrm{a}} \mathrm{P}$ value $<0.05$, Multi-level Mixed effect logistic regression.

Table 4. Related Factors to Primary Sports Headache

\begin{tabular}{llc}
\hline Variant & No. (\%) \\
\hline Sport movement led to PEH & $\begin{array}{l}\text { Resistant movement with the } \\
\text { upper limbs }\end{array}$ & $29(76)$ \\
& $\begin{array}{l}\text { Resistant movement with the } \\
\text { lower limbs }\end{array}$ & $9(24)$ \\
\hline $\begin{array}{l}\text { Position of athlete while } \\
\text { exercising }\end{array}$ & Standing posture & $20(53)$ \\
\hline & Not related to position & $18(47)$ \\
\hline & Neck movement & $29(76)$ \\
\hline heaviness of weights & $28(74)$ \\
& Sexual activity & $16(42)$ \\
& Noise & $15(39)$ \\
\hline & Light & $14(37)$ \\
\hline & Physical activity & $12(32)$ \\
\hline & Thirst & $10(26)$ \\
& Hot weather & $4(11)$ \\
\hline
\end{tabular}

Abbreviation: PEH, primary exercise headache. and benign exertional headache. Aust N Z J Med. 1989;19(5):466-8. [PubMed: 2590098].

24. Imperato J, Burstein J, Edlow JA. Benign exertional headache. Ann Emerg Med. 2003;41(1):98-103. doi: 10.1067/mem.2003.21. [PubMed: 12514689].

25. Lambert RJ, Burnet DL. Prevention of exercise induced migraine by quantitative warm-up. Headache. 1985;25(6):317-9. [PubMed: 4055365].

26. Patel DR, Greydanus DE. Neurologic considerations for adolescent athletes. Adolesc Med. 2002;13(3):569-78. [PubMed: 12270801].

27. Seet RC, Chan YH, Lim EC. Headaches amongst pool players. Headache. 2007;47(2):270-4. doi: 10.1111/j.1526-4610.2006.00692.x. [PubMed: 17300367].

28. Hanashiro S, Takazawa T, Kawase Y, Ikeda K. Prevalence and clinical hallmarks of primary exercise headache in middle-aged Japanese on health check-up. Intern Med. 2015;54(20):2577-81. doi: 10.2169/internalmedicine.54.4926. [PubMed: 26466691].

29. Williams SJ, Nukada H. Sport and exercise headache: Part 2. Diagnosis and classification. Br J Sports Med. 1994;28(2):96-100. [PubMed: 7921916].

30. Noormohammadpour P, Tayyebi F, Mansournia MA, Sharafi E, Kordi R. A concise rehabilitation protocol for sub-acute and chronic non-specific neck pain. J Bodyw Mov Ther. 2017;21(3):472-80. doi: 10.1016/j.jbmt.2016.07.005. [PubMed: 28750953]. 